\title{
A NOTE ON LEFT BIPOTENT NEAR-RINGS
}

\author{
by C. V. L. N. MURTY
}

(Received 23rd August 1983)

In this note it is shown that Theorem 2.8 of [2] is erroneous. Throughout this note near-ring means a right near-ring.

Example. Let $N=\{0,1,2,3,4,5\}$ be the additive group of integers modulo 6 . Define multiplication in $N$ as follows:

\begin{tabular}{c|cccccc}
$\cdot$ & 0 & 1 & 2 & 3 & 4 & 5 \\
\hline 0 & 0 & 0 & 0 & 0 & 0 & 0 \\
1 & 0 & 1 & 4 & 3 & 4 & 1 \\
2 & 0 & 2 & 2 & 0 & 2 & 2 \\
3 & 0 & 3 & 0 & 3 & 0 & 3 \\
4 & 0 & 4 & 4 & 0 & 4 & 4 \\
5 & 0 & 5 & 2 & 3 & 2 & 5
\end{tabular}

Then $N$ is a finite near-ring with $a^{2}=a$ for all $a$ in $N$ (see Clay [1]). Clearly $N$ is a left bipotent $S$-near-ring and 3 is a nonzero left distributive element of $N$. Here $N_{1}=\{0,3\}$ and $N_{2}=\{0,2,4\}$ are the only nontrivial subgroups of $(N,+)$. Further $N=N_{1} \oplus N_{2}$. Clearly $N_{1}$ is a near-field. But $N_{2}$ is not a near-field since $N_{2}$ contains no identity. Thus in this case Theorem 2.8 II of [2] fails.

However if we take the "left identity" instead of "a nonzero distributive element" in Theorem 2.8 II of [2], then the proof is valid.

Acknowledgement. I wish to thank my Research Director Dr Y. V. Reddy for his valuable guidance. Also I wish to express thanks to C.S.I.R. New Delhi for giving me financial assistance.

\section{REFERENCES}

1. J. R. CLAY, The near-rings on groups of low order, Math. Z. 104 (1968), 364-371.

2. J. L. J AT and S. C. Choudhary, On left bipotent near-rings, Proc. Edinburgh Math. Soc. 22 (1979), 99-107.

Department of Mathematics

NAGARJUNA UNIVERSITY

NaGarJunanaGar-522 510

A.P. INDIA 